\title{
Eficácia das limas reciprocantes Reciproc Blue e ProDesign R na remoção de material obturador: Uma revisão integrativa da literatura
}

Efficacy of Reciproc Blue and ProDesign $\mathbb{R}$ reciprocating filles in the removal of filling material: An integrative literature review

Eficacia de los archivos reciprocantes Reciproc Blue y ProDesign R en la eliminación de material de obturación: Una revisión integradora de la literatura

Recebido: 25/04/2021 | Revisado: 03/05/2021 | Aceito: 05/05/2021 | Publicado: 20/05/2021

Andreia Lilian Viana Farias

ORCID: https://orcid.org/0000-0001-5943-3183 Cooperativa de Ensino Superior Politécnico e Universitário, Portugal

E-mail: andreialilianvf@yahoo.com.br

Ana Grasiela da Silva Limoeiro

ORCID: https://orcid.org/0000-0003-4633-720X Faculdade de Ilhéus, Brazil

E-mail: grasielalimoeiro@gmail.com

António Melo Ferraz

ORCID: https://orcid.org/0000-0003-0289-8064 Institute of Health Sciences (IUCS), CESPU, Portugal Institute for Research and Advanced Training in Health Sciences and Technologies (IINFACTS), CESPU, Portugal

E-mail: antonio.ferraz@iucs.cespu.com.br

Wayne Martins Nascimento

ORCID: https://orcid.org/0000-0003-4201-4710 Faculdade São Leopoldo Mandic, Brazil

E-mail: waynemartinsn@gmail.com

Valter Fernandes

ORCID: https://orcid.org/0000-0003-4432-5424 Institute of Health Sciences (IUCS), CESPU, Portugal E-mail: valter.fernandes@iucs.cespu.pt

Carlos Eduardo da Slveira Bueno

ORCID: https://orcid.org/0000-0002-2675-0884 Faculdade São Leopoldo Mandic, Brazil E-mail: carlosesbueno@terra.com.br

Alexandre Sigrist De Martin

ORCID: https://orcid.org/0000-0002-3320-9172 Faculdade São Leopoldo Mandic, Brazil E-mail: a-sigrist@uol.com.br

Paulo Miller

ORCID: https://orcid.org/0000-0001-5500-7603 Institute of Health Sciences (IUCS), CESPU, Portugal Institute for Research and Advanced Training in Health Sciences and Technologies (IINFACTS), CESPU, Portugal E-mail: paulo.miller@iucs.cespu.pt

\begin{abstract}
Resumo
O objetivo do presente estudo foi realizar uma revisão integrativa sobre as propriedades mecânicas dos sistemas reciprocates Reciproc Blue e ProDesign $\mathrm{R}$ e a eficácia desses instrumentos na remoção de material obturador no processo de retratamento endodôntico. Para tanto, foi realizada uma pesquisa na base eletrônica da PUBMED usando as seguintes combinações de termos científicos: Reciproc Blue, Prodesign R, resistance, mechanical properties, thermal treatment e endodontic retreatment. Foram identificados 64 artigos, dos quais 29 foram selecionados para o presente estudo. Os artigos analisados demonstraram a importância do tratamento térmico e de outras características das ligas na rigidez e elasticidade dos instrumentos endodônticos Reciproc Blue e ProDesign R em relação a outros sistemas. Foi observado também que os instrumentos são eficazes na remoção de material obturador do canal radicular, não sendo, no entanto, essa remoção total.
\end{abstract}

Palavra-chave: Reciproc blue; ProDesign R; Propriedades mecânicas; Tratamento térmico; Retratamento endodôntico. 


\begin{abstract}
The aim of the present study was to conduct an integrative review on the mechanical properties of Reciproc Blue and ProDesign $\mathrm{R}$ reciprocating systems and the efficacy of these files in removing root filling materials. The search was carried out on the PUBMED using following combinations of scientific terms: Reciproc Blue, ProDesign R, resistance, mechanical properties, thermal treatment and endodontic retreatment. The research identified 64 articles, of which 29 were selected for the present study. The articles demonstrated the importance of thermomechanical treatment on elasticity and stiffness of Reciproc Blue and ProDesign R endodontic instruments compared to other systems. It was also observed that both files are effective in removing the filling material from the root canal, however both systems failed to remove it completely.
\end{abstract}

Keywords: Reciproc Blue; ProDesign R; Mechanical properties; Thermal treatment; Endodontic retreatment.

\title{
Resumen
}

El objetivo del presente estudio fue realizar una revisión integradora sobre las propiedades mecánicas de los sistemas reciprocantes Reciproc Blue y ProDesign $\mathrm{R}$ y la efectividad de estos instrumentos en la remoción de material de obturación en el proceso de retratamiento endodóntico. Para ello, se realizó una búsqueda en la base electrónica de PUBMED utilizando las siguientes combinaciones de términos científicos: Reciproc Blue, Prodesign R, resistencia, propiedades mecánicas, tratamiento térmico y retratamiento endodóntico. Se identificaron 64 artículos, de los cuales 29 fueron seleccionados para el presente estudio. Los artículos analizados demostraron la importancia del tratamiento térmico y otras características de las aleaciones en la rigidez y elasticidad de los instrumentos de endodoncia Reciproc Blue y ProDesign R en relación con otros sistemas. También se observó que los instrumentos son efectivos para remover el material de obturación del conducto radicular, sin embargo, esta remoción no es total.

Palabras clave: Reciproc blue; Prodesign R; Propiedades mecánicas; Tratamiento térmico; Retratamiento endodóntico.

\section{Introdução}

O principal objetivo da terapia endodôntica é o completo selamento do sistema de canais radiculares, para prevenir e tratar a periodontite apical. Muitos fatores têm sido relacionados com o insucesso neste processo, dos quais podemos realçar a permanência da infecção microbiana dentro dos canais radiculares, a complexidade anatômica, irregularidades na dentina, instrumentação e obturação inadequadas (Roças et al., 2016; Nascimento et al., 2021).

O retratamento endodôntico não cirúrgico é considerado como primeira escolha em casos de falhas de tratamentos prévios (Torabinejad et al., 2009). A remoção completa do material obturador dos canais radiculares é um fator crítico nesse processo (Rios et al., 2014). Diversas técnicas recorrendo ao uso de solventes e instrumentação manual ou mecânica têm sido desenvolvidas para obter melhores resultados na remoção do material obturador (Rodrigues et al., 2016).

A instrumentação endodôntica sofreu uma evolução considerável nos últimos anos nomeadamente no que diz respeito ao tipo de liga e ao tipo de movimentação mecânica dos sistemas. As limas endodônticas são produzidas preferencialmente de ligas de aço-inoxidável e de ligas de níquel-titânio (Ni-Ti). As limas manuais de aço-inoxidável são ainda muito utilizadas na prática endodôntica, mas devido à sua elevada rigidez e pouca elasticidade, apresentam desvantagens, tais como um alto índice de transporte apical, perfurações, desvios e zips (Del Fabbro et al., 2018). Os sistemas de Ni-Ti passaram a ser usados no tratamento endodôntico para reduzir as falhas decorrentes do procedimento endodôntico, podendo ser usados em movimentos rotatórios ou em movimentos reciprocantes.

Os sistemas reciprocantes de Ni-Ti têm sido amplamente utilizados no retratamento endodôntico devido à facilidade de trabalhar em canais curvos e à sua maior segurança e eficiência para remover o material obturador de forma mais rápida do que os instrumentos rotatórios contínuos e manuais (Zuolo et al., 2013). Além disso, os instrumentos reciprocantes apresentam maior resistência a fratura nas tensões flexionais e torcionais em relação a instrumentação rotatória (Ahn et al., 2016).

Recentemente, instrumentos de Ni-Ti com parâmetros térmicos otimizados foram introduzidos para melhorar o desempenho e as propriedades de resistência mecânica em relação aos materiais convencionais (Zupanc et al., 2018). O sistema ProDesign R (PDR- Bassi/Easy Equipamentos Odontológicos, Belo Horizonte, Brasil) é um instrumento de Ni-Ti que apresenta um movimento reciprocante exclusivo e apresenta secção transversal em hélice dupla (forma de S). O processo de fabricação da 
PDR é baseado no tratamento térmico de memória controlada (CM-Wire), proporcionando melhor segurança e eficácia no preparo do canal com um único instrumento (Rodrigues et al., 2016). Os instrumentos Reciproc Blue (RCB- VDW, Munique, Alemanha) são feitos de uma liga de Ni-Ti termicamente tratada e têm sido utilizados em tratamentos de canais radiculares severamente curvos e estreitos (Keskein et al., 2017). A RCB apresenta secção transversal em forma de "S" e liga com “memória de forma" baseado num processo de arrefecimento e de aquecimento que resulta numa camada visível de óxido de titânio na superfície do instrumento, contribuindo para um melhor controle na temperatura de transição (Zupanc et al., 2018).

O preparo do canal radicular e a remoção do material obturador são importantes passos no processo de retratamento endodôntico. Vários estudos têm analisado o desempenho de diferentes instrumentos para essa finalidade (Zuolo et al., 2013; Rios et al., 2014; Limoeiro et al., 2021)

A proposta do presente estudo foi realizar uma revisão integrativa da literatura das propriedades mecânicas dos sistemas reciprocantes PDR e RCB e as suas implicações na eficácia da remoção de material obturador. A hipótese levantada no estudo é de que as propriedades mecânicas dos instrumentos não interferem significativamente na eficácia de remoção de material de obturador no retratamento endodôntico.

\section{Metodologia}

Para seleção dos artigos do estudo foi realizada uma pesquisa bibliográfica na base de dados do PUBMED, utilizando os termos "Reciproc Blue AND resistance", "ProDesign R AND resistance", "Reciproc Blue AND mechanical properties", "ProDesign R AND mechanical properties", "Reciproc Blue AND thermal treatment", "ProDesign R AND thermal treatment", "Reciproc Blue AND endodontic retreatment" e "ProDesign R AND endodontic retreatment".

Os critérios de identificação foram: estudos disponíveis na íntegra, publicações em língua inglesa e publicados até 31 de Dezembro de 2020, que resultaram em 64 artigos. Com a triagem obteve-se 42 artigos, pois os duplicados e os não correspondentes ao período determinado foram removidos. Na etapa de elegibilidade, foi realizada uma leitura do abstract dos 42 artigos, tendo sido excluídos 13 artigos, pois esses não atendiam aos objetivos do estudo, restando assim 29 artigos para etapa de inclusão (Figura 1). 
Figura 1. Fluxograma da seleção dos artigos da revisão.
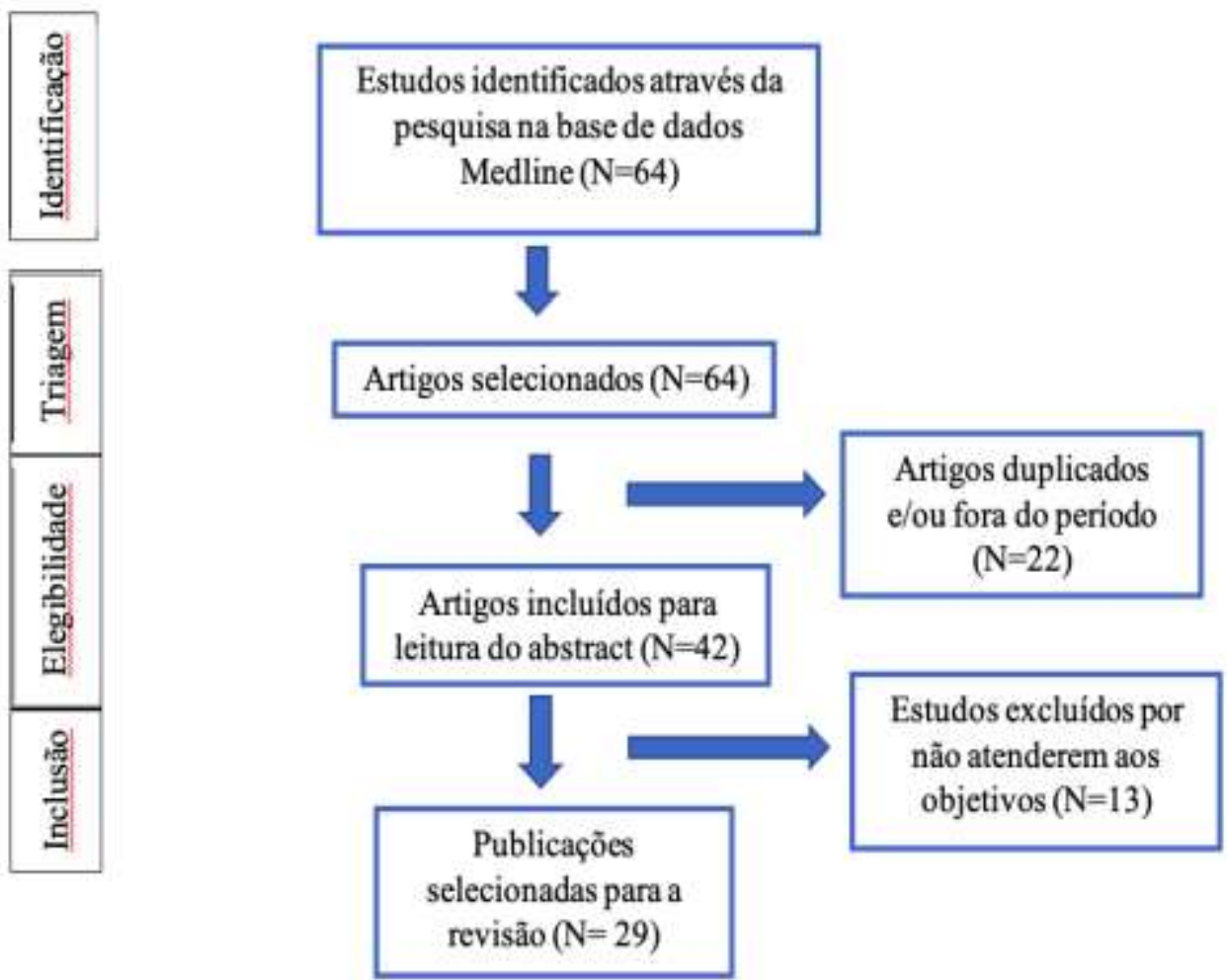

Fonte: Autoria.

Por fim, os artigos selecionados foram catalogados, tendo em vista as seguintes variáveis: autores, ano de publicação, objetivos, resistência à fadiga cíclica flexural e à fadiga torcional das limas ProDesign R e Reciproc Blue, conicidade das limas, ângulos de curvatura dos canais radiculares e eficácia das limas na remoção do material obturador.

Além disso, a discussão foi suportada por outros 24 artigos de relevância, obtidos na base de dados do PUBMED, abordando a temática, tendo por finalidade alargar a fundamentação teórica e que também constam na bibliografia.

\section{Resultados}

Na literatura foram identificados um total de 64 artigos na base de dados do PUBMED. Foi realizada uma avaliação por dois revisores e após a leitura dos títulos e resumos dos artigos, 35 foram excluídos por não se adequarem aos critérios de inclusão (período, duplicidade e relevância). Desta forma, 29 referências foram incluídas neste estudo de revisão (Quadro 1). 
Quadro 1. Dados relevantes dos estudos selecionados para a revisão integrativa.

\begin{tabular}{|c|c|c|c|}
\hline Autores e ano & Proposta & Metodologia & Conclusão \\
\hline $\begin{array}{l}\text { Rodrigues et al. } \\
\text { (2016) }\end{array}$ & $\begin{array}{l}\text { Avaliar a remoção de material } \\
\text { obturador após o uso de } \\
\text { instrumentos CM-wire, M- } \\
\text { wire e Ni-Ti em movimentos } \\
\text { reciprocantes e rotatórios em } \\
\text { canais curvos. }\end{array}$ & $\begin{array}{l}\text { Os canais radiculares foram obturados pela } \\
\text { técnica da condensação lateral com guta-percha } \\
\text { e cimento à base de óxido de zinco e eugenol. O } \\
\text { retratamento foi dividido em } 3 \text { grupos: Reciproc } \\
\text { R25 seguido de Mtwo (40/04) e } \\
\text { ProDesign Logic (50/01), ProDesign R }(25 / 06) \\
\text { seguido de ProDesign Logic (40/05) e } \\
\text { ProDesign Logic (50/01) e Lima K-30 seguido } \\
\text { por K-40 e K-50. }\end{array}$ & $\begin{array}{l}\text { Não foram encontradas diferenças } \\
\text { significativas na remoção do } \\
\text { material obturador nos } 3 \text { grupos. O } \\
\text { uso dos sistemas Mtwo e ProDesign } \\
\text { Logic não melhorou a remoção do } \\
\text { material obturador. }\end{array}$ \\
\hline $\begin{array}{l}\text { De-Deus et al. } \\
\quad(2017)\end{array}$ & $\begin{array}{l}\text { Avaliar a influência do } \\
\text { tratamento térmico Blue na } \\
\text { resistência à flexão e fadiga } \\
\text { cíclica em relação aos } \\
\text { instrumentos convencionais } \\
\text { de M-Wire Reciproc. O } \\
\text { padrão de rugosidade e } \\
\text { microdureza das limas } \\
\text { também foram avaliados }\end{array}$ & $\begin{array}{l}\text { Os instrumentos Reciproc e Reciproc Blue } \\
\text { foram submetidos a testes de resistência à fadiga } \\
\text { cíclica, em um canal artificial de aço inoxidável } \\
\text { com ângulo de } 60^{\circ} \text { e raio de curvatura de } 5 \mathrm{~mm} \text {. } \\
\text { A superfície de fratura de todos os fragmentos } \\
\text { foi examinada através de um microscópio } \\
\text { eletrônico de varredura. }\end{array}$ & $\begin{array}{l}\text { O tratamento térmico Blue mostrou } \\
\text { desempenho geral aprimorado } \\
\text { quando comparado com a liga } \\
\text { convencional } \\
\text { demonstrando aumento da } \\
\text { flexibilidade e resistência à fadiga, e } \\
\text { redução microdureza superficial } \\
\text { enquanto mantém características } \\
\text { semelhantes da superfície. }\end{array}$ \\
\hline $\begin{array}{c}\text { Keskin et al. } \\
\text { (2017) }\end{array}$ & $\begin{array}{l}\text { Comparar a resistência à } \\
\text { fadiga cíclica dos } \\
\text { instrumentos Reciproc Blue } \\
\text { R25, Reciproc R25 e } \\
\text { WaveOne Gold. }\end{array}$ & $\begin{array}{l}\text { Quinze limas Reciproc Blue R25, Reciproc R } 25 \\
\text { e WaveOne Gold primary foram acionadas em } \\
\text { um dispositivo de teste de fadiga cíclico } \\
\text { dinâmico, com ângulo de curvatura } 60^{\circ} \text { e raio de } \\
5 \mathrm{~mm} \text { de curvatura. Todos os instrumentos } \\
\text { foram operados até a fratura, e o tempo até a } \\
\text { fratura e o comprimento dos fragmentos } \\
\text { fraturados foram registrados. }\end{array}$ & $\begin{array}{l}\text { Os instrumentos Reciproc Blue } \\
\text { apresentaram resistência à fadiga } \\
\text { cíclica significativamente maior } \\
\text { comparado aos sistemas WaveOne } \\
\text { Gold e Reciproc. }\end{array}$ \\
\hline $\begin{array}{l}\text { Topçuoğlu \& } \\
\text { Topçuoğlu } \\
\text { (2017) }\end{array}$ & $\begin{array}{l}\text { Avaliar a resistência à fadiga } \\
\text { cíclica das limas Reciproc R25 } \\
\text { e R40, e Reciproc Blue R25 e } \\
\text { R40 em canal artificial em } \\
\text { forma de S. }\end{array}$ & $\begin{array}{l}\text { O teste de fadiga cíclica foi realizado em canais } \\
\text { artificiais em forma de } \mathrm{S} \text {, apresentando duas } \\
\text { curvas: } 60^{\circ} \text { de ângulo de curvatura, } 5 \mathrm{~mm} \text { de raio } \\
\text { a } 8 \mathrm{~mm} \text { da ponta do instrumento (curvatura } \\
\text { apical); e } 70^{\circ} \text { de ângulo de curvatura, } 2 \mathrm{~mm} \text { de } \\
\text { raio e centro a } 2 \mathrm{~mm} \text { da ponta (curvatura } \\
\text { coronal). Os instrumentos foram ativados por } \\
\text { motor controlado por torque, utilizando o } \\
\text { programa Reciproc ALL até a detecção da } \\
\text { fratura. }\end{array}$ & $\begin{array}{l}\text { Os instrumentos Reciproc Blue R25 } \\
\text { e R40 mostraram maior resistência à } \\
\text { fratura cíclica comparado aos } \\
\text { instrumentos Reciproc R25 e R40 } \\
\text { em um canal em forma de S. }\end{array}$ \\
\hline $\begin{array}{l}\text { Gündoğar \& } \\
\text { Özyürek } \\
\text { (2017) }\end{array}$ & $\begin{array}{l}\text { Comparar a resistência à } \\
\text { fadiga cíclica dos sistemas } \\
\text { Reciproc Blue, HyFlex EDM, } \\
\text { WaveOne Gold e OneShape. }\end{array}$ & $\begin{array}{l}\text { As limas Reciproc Blue }(25 / 08) \text {, HyFlex EDM } \\
(25 / . \sim) \text {, WaveOne Gold }(25 / 07) \text { e OneShape } \\
(25 / 06) \text { foram submetidas ao teste de resistência } \\
\text { à fadiga cíclica, em canais artificiais de aço } \\
\text { inoxidável com ângulo de } 60^{\circ} \text {, raio de curvatura } \\
\text { de } 5 \mathrm{~mm} \text { e diâmetro interno de } 5 \mathrm{~mm} \text {. }\end{array}$ & $\begin{array}{l}\text { A resistência à fadiga cíclica da } \\
\text { HyFlex EDM foi maior comparado } \\
\text { aos instrumentos OneShape, } \\
\text { Reciproc Blue e WaveOne Gold. }\end{array}$ \\
\hline $\begin{array}{l}\text { Silva et al } \\
(2018 a)\end{array}$ & $\begin{array}{l}\text { O objetivo do estudo foi } \\
\text { avaliar a influência do } \\
\text { tratamento térmico BLUE na } \\
\text { resistência à torção de limas } \\
\text { Reciproc M-Wire }\end{array}$ & $\begin{array}{l}\text { Reciproc (25/08) } \\
\text { Reciproc Blue (25/08) } \\
\text { O teste de resistência torcional foi realizado em } \\
\text { dispositivo personalizado, aplicando uma carga } \\
\text { torcional até a fratura do instrumento. Todos os } \\
\text { instrumentos foram girados no sentido anti- } \\
\text { horário a uma velocidade de } 2 \mathrm{rpm} \text {. }\end{array}$ & - \\
\hline $\begin{array}{l}\text { Özyürek et al. } \\
\qquad(2018)\end{array}$ & $\begin{array}{l}\text { Comparar a resistência à } \\
\text { fadiga cíclica dos } \\
\text { instrumentos HyFlex EDM, } \\
\text { WaveOne Gold, Reciproc } \\
\text { Blue e 2Shape. }\end{array}$ & $\begin{array}{l}\text { Os instrumentos HyFlex EDM, WaveOne Gold, } \\
\text { Reciproc Blue e } 2 \text { Shape foram acionados em } \\
\text { canais artificiais de aço inoxidável com diâmetro } \\
\text { interno de } 1,5 \mathrm{~mm} \text {, ângulos de curvatura de } 45^{\circ} \\
\text { e } 90^{\circ} \text { e um raio de curvatura de } 5 \mathrm{~mm} \text {. O tempo } \\
\text { para fratura e o número de ciclos até a fratura } \\
\text { foram medidos. }\end{array}$ & $\begin{array}{l}\text { As limas Reciproc Blue } \\
\text { apresentaram resistência à fadiga } \\
\text { cíclica estatisticamente maior em } \\
\text { canais artificiais com } 45^{\circ} \text { e } 90^{\circ} \\
\text { comparado a outras limas avaliadas. }\end{array}$ \\
\hline
\end{tabular}




\begin{tabular}{|c|c|c|c|}
\hline $\begin{array}{l}\text { Plotino et al. } \\
\qquad(2018)\end{array}$ & $\begin{array}{l}\text { Investigar o impacto da } \\
\text { temperatura na fadiga cíclica } \\
\text { em sistemas de Ni-Ti }\end{array}$ & $\begin{array}{l}\text { O teste de fadiga cíclica dos instrumentos } \\
\text { Reciproc e Reciproc Blue instrumentos NiTi foi } \\
\text { realizado em um canal artificial de aço } \\
\text { inoxidável. Durante o experimento, } 4 \\
\text { temperaturas diferentes }\left(0^{\circ} \mathrm{C}, 20^{\circ} \mathrm{C}, 35^{\circ} \mathrm{C} \text { e }\right. \\
39 \text { C) foram utilizadas em um banho } \\
\text { termostático controlado por controles } \\
\text { eletrônicos e infravermelhos. Oitenta } \\
\text { instrumentos Reciproc Blue R } 25 \text { e } 80 \text { Reciproc } \\
\text { R25 foram alternados no canal artificial até a } \\
\text { fratura ocorrer, e o tempo de fratura e o } \\
\text { comprimento do fragmento fraturado foram } \\
\text { registrados. A temperatura de transformação de } \\
\text { fase para os } 3 \text { instrumentos de cada tipo foi } \\
\text { analisada por calorimetria diferencial de } \\
\text { varredura. }\end{array}$ & $\begin{array}{l}\mathrm{O} \text { tratamento Blue aumenta } \\
\text { significativamente a resistência à } \\
\text { fadiga cíclica das limas Reciproc em } \\
\text { comparação com a liga M-Wire. A } \\
\text { temperatura afeta significativamente } \\
\text { a vida útil das limas NiTi. Quando a } \\
\text { temperatura ambiente aumenta entre } \\
0^{\circ} \mathrm{C} \text { e } 35^{\circ} \mathrm{C} \text {, a resistência à fadiga } \\
\text { das limas diminui estatisticamente. }\end{array}$ \\
\hline $\begin{array}{l}\text { Topçuoğlu et al. } \\
\text { (2018) }\end{array}$ & $\begin{array}{l}\text { Comparar a resistência à } \\
\text { fadiga cíclica dos sistemas } \\
\text { Reciproc Blue, WaveOne } \\
\text { Gold e SmartTrack em canais } \\
\text { artificiais curvos. }\end{array}$ & $\begin{array}{l}\text { Noventa limas Reciproc Blue R25, WaveOne } \\
\text { Gold Primary e SmartTrack X1 foram testados } \\
\text { em canais artificiais com ângulos de curvatura } \\
\text { de } 45^{\circ} \text { e } 60^{\circ} \text {. A resistência à fratura cíclica foi } \\
\text { determinada registrando-se o tempo de fratura } \\
\text { nos canais artificiais. }\end{array}$ & $\begin{array}{l}\text { Os resultados do presente estudo } \\
\text { mostraram que os sistemas Reciproc } \\
\text { Blue e SmartTrack exibiram maior } \\
\text { resistência à fratura cíclica } \\
\text { comparado aos instrumentos } \\
\text { WaveOne Gold apenas em canais } \\
\text { com ângulo de curvatura de } 60^{\circ} \text {. }\end{array}$ \\
\hline $\begin{array}{l}\text { Iacono et al. } \\
\text { (2019) }\end{array}$ & $\begin{array}{l}\text { Analisar o impacto de } \\
\text { diferentes tipos movimentos } \\
\text { na fadiga cíclica de quatro } \\
\text { instrumentos reciprocantes de } \\
\text { Ni-Ti }\end{array}$ & $\begin{array}{l}\text { A resistência à fadiga cíclica de } 160 \\
\text { instrumentos foi avaliada em um canal artificial } \\
\text { de aço inoxidável com ângulo de } 90^{\circ} \text { e raio de } \\
\text { curvatura de } 5 \mathrm{~mm} \text {. WaveOne, WaveOne Gold, } \\
\text { Reciproc e Reciproc Blue foram testados com } \\
\text { dois movimentos diferentes: Motor endodôntico } \\
\text { X-Smart Plus ou contra-ângulo } 4: 1 \text { com } \\
\text { movimento experimental (EVO) com diferentes } \\
\text { ângulos de rotação e baseado em uma aceleração } \\
\text { senoidal. Os movimentos com X-Smart Plus e } \\
\text { EVO foram gravados e analisados em } \\
\text { velocidade reduzida com o software VLC Media } \\
\text { Player para uma análise mais precisa. A meia- } \\
\text { vida média, os parâmetros beta e eta Weibull } \\
\text { foram determinados e comparados. }\end{array}$ & $\begin{array}{l}\text { Reciproc Blue resultou nos } \\
\text { instrumentos mais resistentes com } \\
\text { EVO ou X-Smart. O WaveOne Gold } \\
\text { durou significativamente mais do } \\
\text { que o WaveOne com EVO, } \\
\text { enquanto nenhuma diferença } \\
\text { significativa foi encontrada com o } \\
\text { X-Smart. Considerando NCF, } \\
\text { Reciproc, WaveOne Gold e } \\
\text { Reciproc Blue duraram } \\
\text { significativamente mais com EVO. } \\
\text { Descobriu-se que o movimento } \\
\text { experimental teve um impacto } \\
\text { positivo na vida útil de fadiga de } \\
\text { instrumentos alternativos. }\end{array}$ \\
\hline $\begin{array}{l}\text { Al-Obaida et al. } \\
\text { (2019) }\end{array}$ & $\begin{array}{l}\text { Comparar a resistência à } \\
\text { fadiga cíclica de } 5 \text { limas de Ni- } \\
\text { Ti termicamente tratadas, que } \\
\text { apresentam o mesmo tamanho } \\
\text { de ponta e diferentes seções } \\
\text { transversais. }\end{array}$ & $\begin{array}{l}\text { As limas WaveOne, WaveOne Gold, Reciproc, } \\
\text { Reciproc Blue e TF foram avaliadas. A } \\
\text { resistência à fadiga cíclica foi comparada entre } \\
\text { os grupos determinando o tempo necessário para } \\
\text { a fratura e o número de ciclos até a falha em um } \\
\text { dispositivo de teste de fadiga cíclica com } 2 \\
\text { curvaturas diferentes, a primeira com um raio de } \\
\text { curvatura de } 5 \mathrm{~mm} \text { e um ângulo de } 60^{\circ} \text { e a } \\
\text { segunda com curvatura dupla, curvatura coronal } \\
\text { de ângulo de } 60^{\circ} \text { e raio de } 5 \mathrm{~mm} \text {, e curvatura } \\
\text { apical de ângulo de } 70^{\circ} \text { e raio de } 2 \mathrm{~mm} \text {. A } \\
\text { avaliação por microscopia eletrônica de } \\
\text { varredura foi realizada nos locais de fratura para } \\
\text { investigar os tipos de fratura. }\end{array}$ & $\begin{array}{l}\text { As limas Reciproc Blue exibiram } \\
\text { resistência à fadiga cíclica } \\
\text { significativamente maior em } \\
\text { comparação com outras limas } \\
\text { testadas em um canal artificial em } \\
\text { forma de S. }\end{array}$ \\
\hline $\begin{array}{l}\text { Almeida et al. } \\
\qquad(2019)\end{array}$ & $\begin{array}{l}\text { Comparar as temperaturas de } \\
\text { transformação martensítica e o } \\
\text { comportamento da resistência } \\
\text { à torção dos instrumentos } \\
\text { Reciproc e Reciproc blue. }\end{array}$ & $\begin{array}{l}\text { As temperaturas de transformação dos } \\
\text { instrumentos Reciproc Blue e Reciproc R25 } \\
\text { foram determinadas por calorimetria de } \\
\text { varredura diferencial (DSC - DSC } 60 \text {, } \\
\text { Shimadzu, Kyoto, Japão). Três amostras com } \\
\text { aproximadamente } 20 \mathrm{mg} \text { de cada sistema foram } \\
\text { resfriadas a }-120{ }^{\circ} \mathrm{C} \text {, aquecidas a } 100^{\circ} \mathrm{C} \text { e então } \\
\text { resfriadas a }-100{ }^{\circ} \mathrm{C} \text { a uma taxa de } 10^{\circ} \mathrm{C} \text { min- } 1 . \\
\text { Os valores máximos de torque e deflexão }\end{array}$ & $\begin{array}{l}\text { Os instrumentos Reciproc e } \\
\text { Reciproc blue são compostos de uma } \\
\text { mistura de fase } \mathrm{R} \text { e austenita. Os } \\
\text { instrumentos Reciproc Blue } \\
\text { apresentaram um ângulo de rotação } \\
\text { maior para fraturar, mas um torque } \\
\text { mais baixo para falha do que os } \\
\text { instrumentos Reciproc. }\end{array}$ \\
\hline
\end{tabular}




\begin{tabular}{|c|c|c|c|}
\hline & & $\begin{array}{l}\text { angular até a fratura de novos instrumentos }(\mathrm{n}= \\
\text { 10) foram medidos de acordo com a ISO } 3630- \\
\text { 1. }\end{array}$ & \\
\hline $\begin{array}{c}\text { Klymus et al. } \\
\text { (2019) }\end{array}$ & $\begin{array}{l}\text { Avaliar o impacto da } \\
\text { temperatura corporal na } \\
\text { resistência à fadiga cíclica das } \\
\text { limas Reciproc Blue R25 } \\
\text { (VDW, Munique, Alemanha), } \\
\text { X1 Blue File 25 (MK Life } \\
\text { Medical and Dental Products, } \\
\text { Porto Alegre, Brasil) e } \\
\text { WaveOne Gold Primary } \\
\text { (Dentsply Maillefer, } \\
\text { Ballaigues, Suíça). }\end{array}$ & $\begin{array}{l}\text { Foram utilizados } 20 \text { instrumentos de cada } \\
\text { sistema: Reciproc Blue R25 (25.08), x1 Blue } \\
\text { File (25.06) e Wave One Gold Primary (25.07). } \\
\text { Os testes de fadiga cíclica foram realizados à } \\
\text { temperatura ambiente }\left(20^{\circ} \pm 1{ }^{\circ} \mathrm{C}\right) \text { e à } \\
\text { temperatura corporal }\left(37^{\circ} \mathrm{C} \pm 1{ }^{\circ} \mathrm{C}\right) \text {. Os } \\
\text { instrumentais foram acionados em movimento } \\
\text { reciprocante até a fratura em canal artificial de } \\
\text { aço inoxidável com ângulo de } 60^{\circ} \text { e raio de } \\
\text { curvatura de } 5 \mathrm{~mm} \text {. O tempo até a fratura (TTF) } \\
\text { foi registrado. Além disso, o número de ciclos } \\
\text { até a fratura (NCF) foi calculado. }\end{array}$ & $\begin{array}{l}\text { A temperatura corporal causou uma } \\
\text { redução acentuada da resistência à } \\
\text { fadiga cíclica para todos os } \\
\text { instrumentos testados. Os sistemas } \\
\text { Reciproc Blue e X1 Blue File } \\
\text { apresentaram resultados } \\
\text { semelhantes nas duas temperaturas } \\
\text { testadas. No entanto, o Wave One } \\
\text { Gold apresentou a menor redução } \\
\text { percentual na resistência à fadiga à } \\
\text { temperatura corporal. }\end{array}$ \\
\hline $\begin{array}{c}\text { Inan et al. } \\
\text { (2019) }\end{array}$ & $\begin{array}{l}\text { Avaliar a resistência à fadiga } \\
\text { cíclica das limas } \\
\text { Reciproc e Reciproc Blue em } \\
\text { canais de curvatura apical } \\
\text { graves em tempertura } \\
\text { intracanal }\end{array}$ & $\begin{array}{l}\text { Dezoito instrumentos Reciproc R } 25 \text { e Reciproc } \\
\text { Blue foram testados em um dispositivo de fadiga } \\
\text { cíclica à temperatura corporal }\left(37^{\circ} \mathrm{C}\right) \text { usando } \\
\text { um bloco de aço inoxidável com um canal } \\
\text { artificial com ângulo de curvatura de } 90^{\circ} \text { e raio } \\
\text { de curvatura de } 2 \text { milímetros. O número de } \\
\text { ciclos até a fratura (NCF) foi calculado, e os } \\
\text { instrumentos foram examinados por calorimetria } \\
\text { exploratória diferencial (DSC). }\end{array}$ & 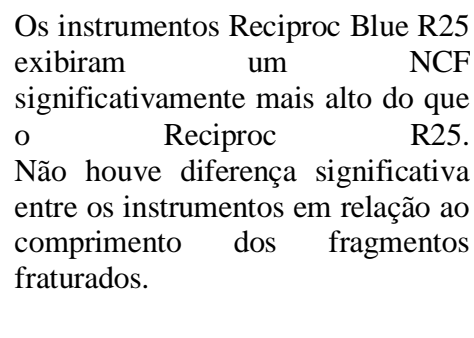 \\
\hline $\begin{array}{l}\text { Silva et al. } \\
\text { (2019) }\end{array}$ & $\begin{array}{l}\text { Avaliar a resistência à fadiga } \\
\text { cíclica do Reciproc e Reciproc } \\
\text { Blue acionados por dois tipos } \\
\text { de motores: Direct@ Contra- } \\
\text { ângulo ou motor elétrico } \\
\text { controlado por torque. }\end{array}$ & $\begin{array}{l}\text { Trinta instrumentos Reciproc R } 25 \text { e } 30 \text { Reciproc } \\
\text { Blue R25 foram submetidas ao teste de } \\
\text { resistência à fadiga cíclica em um canal artificial } \\
\text { de aço inoxidável com ângulo de } 60^{\circ} \text { e raio de } \\
\text { curvatura de } 5 \mathrm{~mm} \text {. Os instrumentos Reciproc e } \\
\text { Reciproc Blue foram ativados com uma peça de } \\
\text { mão de redução } 6: 1 \text { alimentada por um motor } \\
\text { controlado por torque usando o programa } \\
\text { predefinido "RECIPROC ALL", com contra- } \\
\text { ângulo Reciproc Direct } 囚 \text { alimentado por um } \\
\text { motor pneumático comum ou com Contra- } \\
\text { ângulo Direct® acionado por motor elétrico. A } \\
\text { superfície de fratura de todos os fragmentos fo } \\
\text { examinada com um microscópio eletrônico de } \\
\text { varredura. }\end{array}$ & $\begin{array}{l}\text { Os instrumentos Reciproc Blue } \\
\text { apresentaram melhor desempenho } \\
\text { em relação à resistência à fadiga } \\
\text { cíclica quando comparados aos } \\
\text { instrumentos Reciproc. Os } \\
\text { instrumentos acionados pelo contra- } \\
\text { ângulo Reciproc Direct }{ }^{\circledR} \\
\text { apresentaram maior fadiga cíclica e } \\
\text { número de ciclos até a fratura que os } \\
\text { instrumentos acionados por um } \\
\text { motor elétrico controlado por } \\
\text { torque. }\end{array}$ \\
\hline $\begin{array}{l}\text { Generali et al. } \\
\text { (2020) }\end{array}$ & $\begin{array}{l}\text { Comparar as propriedades } \\
\text { mecânicas e metalúrgicas dos } \\
\text { instrumentos Reciproc e } \\
\text { Reciproc Blue. }\end{array}$ & $\begin{array}{l}\text { Um total de } 120 \text { instrumentos R25 Reciproc } \\
\text { Blue e R25 Reciproc foram utilizados. As } \\
\text { características morfológicas, químicas, } \\
\text { mecânicas, térmicas e de composição de fase } \\
\text { foram investigadas por microscopia eletrônica } \\
\text { de varredura (FEG-SEM) com espectroscopia de } \\
\text { energia dispersiva de raios-X (EDS), análise de } \\
\text { feixe de íons focado (FIB), micro - } \\
\text { Espectroscopia Raman, metalografia FEG-SEM } \\
\text { difração de raios X (XRD), calorimetria } \\
\text { diferencial de varredura (DSC) e testes de } \\
\text { indentação. A degradação induzida pelo uso foi } \\
\text { avaliada. Dez instrumentos novos e dez usados } \\
\text { por tipo foram executados até que a fratura } \\
\text { ocorresse em um canal artificial de aço } \\
\text { inoxidável (ângulo de curvatura de } 60 \text {, raio de } \\
4 \text { mm). O tempo de fratura e o comprimento do } \\
\text { fragmento fraturado foram registrados. Torque e } \\
\text { ângulo de rotação na falha de dez instrumentos } \\
\text { novos e dez usados para cada tipo foram } \\
\text { medidos de acordo com a ISO } 3630-1 \text {. A } \\
\text { superfície de fratura de cada fragmento foi } \\
\text { examinada. }\end{array}$ & $\begin{array}{l}\text { O tratamento térmico do Reciproc } \\
\text { Blue foi associado a uma estrutura } \\
\text { mais fina e com grãos menores que } \\
\text { o Reciproc, o que aumentou sua } \\
\text { resistência à fratura e também foi } \\
\text { responsável por sua menor dureza e } \\
\text { menor módulo de elasticidade. } \\
\text { Ambos os instrumentos foram } \\
\text { seguros durante o uso ex vivo em } \\
\text { canais severamente curvados. }\end{array}$ \\
\hline
\end{tabular}




\begin{tabular}{|c|c|c|c|}
\hline $\begin{array}{l}\text { Silva et al. } \\
\text { (2018b) }\end{array}$ & $\begin{array}{l}\text { Avaliar a fadiga cíclica e } \\
\text { resistência à torção dos } \\
\text { sistemas ProDesign R, } \\
\text { Reciproc Blue e WaveOne } \\
\text { Gold em canais severamente } \\
\text { curvos. }\end{array}$ & $\begin{array}{l}\text { Os instrumentos ProDesign R (25/06), Reciproc } \\
\text { Blue }(25 / 08) \text { e WaveOne Gold (25/07) foram } \\
\text { submetidos ao teste de fadiga cíclica em canais } \\
\text { simulados severamente curvos, com ângulo de } \\
\text { curvatura de } 80^{\circ} \text { e } 3 \text { mm de raio de curvatura. Os } \\
\text { instrumentos foram acionados de acordo com as } \\
\text { recomendações, até a ocorrência de fratura. }\end{array}$ & $\begin{array}{l}\text { ProDesign } \mathrm{R} \text { apresentou a maior } \\
\text { resistência à fadiga cíclica em canais } \\
\text { severamente curvos quando } \\
\text { comparado com Reciproc Blue e } \\
\text { Wave One Gold. No entanto, } \\
\text { Reciproc Blue apresentou a maior } \\
\text { resistência à torção geral e maior } \\
\text { ângulo de rotação para fratura } \\
\text { quando comparado ao ProDesign R. }\end{array}$ \\
\hline $\begin{array}{l}\text { Alcalde et al. } \\
\qquad(2018)\end{array}$ & $\begin{array}{l}\text { O objetivo deste estudo foi } \\
\text { avaliar a resistência à fadiga } \\
\text { cíclica e torcional dos sistemas } \\
\text { reciprocantes Reciproc Blue } \\
\text { 25.08, ProDesign R 25.06 e } \\
\text { WaveOne Gold 25.07. }\end{array}$ & $\begin{array}{l}\text { Instrumentos Reciproc Blue R25 (25.08), } \\
\text { ProDesign R (25.06) e WaveOne Gold (25.07) } \\
\text { foram submetidos ao teste de resistência à fadiga } \\
\text { cíclica em um canal artificial de aço inoxidável } \\
\text { com ângulo de curvatura de } 60^{\circ} \text { e raio de } 5 \mathrm{~mm} \\
\text { localizado a } 5 \mathrm{~mm} \text { da ponta. A superfície } \\
\text { fraturada de cada fragmento foi avaliada por } \\
\text { meio de microscopia eletrônica de varredura } \\
\text { (MEV). Além disso, um exame complementar } \\
\text { foi realizado para medir a área transversal de } \\
\text { cada instrumento a } 3 \text { e } 5 \mathrm{~mm} \text { da ponta. }\end{array}$ & $\begin{array}{l}\text { Prodesign } \mathrm{R} \text { apresentou maior } \\
\text { resistência à fadiga cíclica e rotação } \\
\text { angular até a fratura em comparação } \\
\text { com Reciproc Blue e WaveOne } \\
\text { Gold. Reciproc Blue e WaveOne } \\
\text { Gold apresentaram maior resistência } \\
\text { à torção do que Prodesign } \mathrm{R} \text {. }\end{array}$ \\
\hline $\begin{array}{l}\text { De-Menezes et } \\
\text { al. }(2017)\end{array}$ & $\begin{array}{l}\text { Avaliar o tempo de } \\
\text { instrumentação e a resistência } \\
\text { à fadiga cíclica das limas } \\
\text { WaveOne Gold, ProDesign R } \\
\text { e ProDesign Logic. }\end{array}$ & $\begin{array}{l}\text { As limas ProDesign Logic } 25.06 \text { foram } \\
\text { utilizadas em rotação contínua após a preparação } \\
\text { do glide path. As limas WaveOne Gold } 25.07 \text { e } \\
\text { ProDesign R } 25.06 \text { foram usadas em movimento } \\
\text { reciprocante. Cada lima instrumentou } 3 \text { canais } \\
\text { artificiais padronizados. O tempo médio, o } \\
\text { número de ciclos (NCI) e a resistência à fadiga } \\
\text { cíclica de cada lima foram determinados através } \\
\text { do número de ciclos até a falha (NCF) em um } \\
\text { dispositivo de aço inoxidável. A quantidade total } \\
\text { de ciclos até a fratura também foi calculada (NCI } \\
+\mathrm{NCF} \text { ). }\end{array}$ & $\begin{array}{l}\text { O uso do movimento rotatório } \\
\text { contínuo com a lima ProDesign } \\
\text { Logic em canais com glide path } \\
\text { levou a um menor tempo de } \\
\text { instrumentação. A resistência à } \\
\text { fadiga cíclica dos instrumentos } \\
\text { ProDesign R e Logic foi superior ao } \\
\text { WaveOne Gold. O tratamento } \\
\text { térmico da liga do instrumento, sua } \\
\text { seção transversal e a trajetória de } \\
\text { deslizamento parecem influenciar a } \\
\text { resistência à fadiga cíclica. }\end{array}$ \\
\hline $\begin{array}{l}\text { Alcalde et al. } \\
\qquad(2017)\end{array}$ & $\begin{array}{l}\text { Analisar a resistência à fadiga } \\
\text { cíclica e torcional dos sistemas } \\
\text { ProDesign R, Reciproc R25 e } \\
\text { Unicone L25. }\end{array}$ & $\begin{array}{l}\text { Vinte instrumentos de cada sistema ProDesign R } \\
\text { (25.06), Reciproc R25 (25.08) e Unicone L } 25 \\
\text { (25.06) foram utilizados. A resistência à fadiga } \\
\text { cíclica foi testada medindo o tempo de ruptura } \\
\text { em um canal artificial de aço inoxidável com } \\
\text { ângulo de } 60^{\circ} \text { e raio de curvatura de } 5 \mathrm{~mm} \text {. O } \\
\text { torque e o ângulo de rotação na fratura dos } \\
\text { instrumentos nos } 3 \mathrm{~mm} \text { da porção da ponta } \\
\text { foram medidos durante o teste de torção de } \\
\text { acordo com a ISO } 3630-1 \text {. A superfície fraturada } \\
\text { de cada fragmento foi examinada por } \\
\text { microscopia eletrônica de varredura. }\end{array}$ & $\begin{array}{l}\text { ProDesign } \mathrm{R} \text { apresentou maior } \\
\text { resistência à fadiga cíclica e rotação } \\
\text { angular para falha em comparação } \\
\text { com Reciproc e Unicone. No } \\
\text { entanto, Reciproc mostrou maior } \\
\text { resistência à torção até a fratura. }\end{array}$ \\
\hline $\begin{array}{l}\text { Silva et al. } \\
\text { (2016) }\end{array}$ & $\begin{array}{l}\text { Avaliar a resistência à } \\
\text { flexibilidade e à fadiga cíclica } \\
\text { do instrumento reciprocante } \\
\text { ProDesign R. }\end{array}$ & $\begin{array}{l}\text { A resistência à flexão dos instrumentos } \\
\text { ProDesign } \mathrm{R} \text { (25.06), ProDesign R não tratada } \\
(25.06) \text {, Reciproc R25 (25.08), WaveOne } \\
\text { (25.08) foram realizada utilizando-se uma } \\
\text { máquina universal de ensaios. A resistência à } \\
\text { fadiga cíclica foi testada medindo o número de } \\
\text { ciclos até a fratura em um canal artificial de aço } \\
\text { inoxidável. A análise de microscopia eletrônica } \\
\text { de varredura foi realizada para determinar o } \\
\text { modo de fratura e possíveis deformações na } \\
\text { haste helicoidal. }\end{array}$ & $\begin{array}{l}\mathrm{O} \text { instrumento reciprocante } \\
\text { termicamente tratado ProDesign } \mathrm{R} \\
\text { tem maior resistência à fadiga cíclica } \\
\text { do que os instrumentos ProDesign } \mathrm{R} \\
\text { não tratados, Reciproc e WaveOne. } \\
\text { ProDesign } \mathrm{R} \text { e Reciproc foram } \\
\text { significativamente mais flexíveis do } \\
\text { que os instrumentos ProDesign } \mathrm{R} e \\
\text { WaveOne não tratados. }\end{array}$ \\
\hline $\begin{array}{l}\text { Bago et al. } \\
\text { (2019) }\end{array}$ & $\begin{array}{l}\text { Comparar os sistemas } \\
\text { Reciproc Blue, Reciproc e } \\
\text { ProTaper Universal em } \\
\text { retratamento de canais ovais } \\
\text { retos. }\end{array}$ & $\begin{array}{l}\text { Dentes extraídos com canais ovais retos únicos } \\
\text { foram instrumentados com o sistema ProTaper } \\
\text { Next e cimento à base de guta-percha e resina } \\
\text { epóxi usando a técnica de condensação lateral } \\
\text { fria. As amostras foram divididas em três grupos } \\
\text { de retratamento: Reciproc Blue R40, Reciproc } \\
\text { R40 ou ProTaper Universal. }\end{array}$ & $\begin{array}{l}\text { O Sistema Reciproc removeu } \\
\text { significativamente mais material } \\
\text { obturador em relação aos } \\
\text { instrumentos ProTaper e Reciproc } \\
\text { Blue. Os sistemas ProTaper e } \\
\text { Reciproc Blue apresentaram o } \\
\text { mesmo volume residual. }\end{array}$ \\
\hline
\end{tabular}




\begin{tabular}{|c|c|c|c|}
\hline $\begin{array}{l}\text { Borges et al. } \\
\text { (2019) }\end{array}$ & $\begin{array}{l}\text { Avaliar três sistemas } \\
\text { reciprocantes e métodos de } \\
\text { limpeza complementares na } \\
\text { remoção do material obturador } \\
\text { durante o retratamento de } \\
\text { canais achatados. }\end{array}$ & $\begin{array}{l}\text { Incisivos inferiores extraídos foram } \\
\text { instrumentados até o tamanho } 25.08 \text {, e obturados } \\
\text { com a técnica de cone único e cimento AH-Plus. } \\
\text { Posteriormente os dentes foram retratados com } \\
\text { Reciproc Blue R25 (25.08) e R40 (40.06), ou } \\
\text { ProDesign R (25.06) e (35.05) } \\
\text { Ou WaveOne Gold primary (25.07) e } \\
\text { medium(35.06). } \\
\text { Após o retratamento, os dentes foram divididos } \\
\text { em três grupos para tratamento complementar: } \\
\text { XP-Endo Shaper (30.01); Irrigação ultrassônica } \\
\text { passiva (PUI) ou instrumentação oscilatória } \\
\left(60^{\circ}\right) \text { com lima H \#30. }\end{array}$ & $\begin{array}{l}\text { Nenhum dos sistemas removeu } \\
\text { completamente o material obturador } \\
\text { e não houve diferença estatística } \\
\text { entre os grupos, independentemente } \\
\text { do tamanho do instrumento. A } \\
\text { limpeza complementar aumentou a } \\
\text { remoção do material residual, sendo } \\
\text { o sistema XP-Endo Shaper mais } \\
\text { eficaz significativamente que a lima } \\
\text { H e PUI. }\end{array}$ \\
\hline $\begin{array}{l}\text { De-Deus et al. } \\
\qquad(2019)\end{array}$ & $\begin{array}{l}\text { Comparar a eficiência das } \\
\text { limas Reciproc e Reciproc } \\
\text { Blue na remoção de material } \\
\text { obturador e na capacidade de } \\
\text { recuperação da patência } \\
\text { apical. }\end{array}$ & $\begin{array}{l}\text { Dentes com presença de canal único ovalado } \\
\text { foram instrumentados com a Reciproc R } 25 \text { e } \\
\text { obturados com guta-percha e cimento AH-Plus } \\
\text { usando a técnica de condensação de onda } \\
\text { contínua. } \\
\text { O processo de retratamento iniciou-se com o uso } \\
\text { das brocas Gates-Glidden, e sem seguida foram } \\
\text { instrumentados com Reciproc R } 25 \text { seguido de } \\
\text { Reciproc R40, ou Reciproc Blue R } 25 \text { seguido de } \\
\text { Reciproc Blue R40. }\end{array}$ & $\begin{array}{l}\text { Não foi observada diferença } \\
\text { significativa entre os instrumentos } \\
\text { na remoção de material obturador. O } \\
\text { alargamento apical do tamanho } 25 \\
\text { para } 40 \text { melhorou significativamente } \\
\text { a remoção do material obturador. }\end{array}$ \\
\hline $\begin{array}{l}\text { De-Deus et al. } \\
\quad(2019 b)\end{array}$ & $\begin{array}{l}\text { Avaliar a eficácia do XP-endo } \\
\text { Shaper na remoção de } \\
\text { material obturador de canais } \\
\text { ovais, usando os instrumentos } \\
\text { Reciproc e Reciproc Blue } \\
\text { como referência. }\end{array}$ & $\begin{array}{l}\text { Trinta incisivos inferiores com canais ovais } \\
\text { foram instrumentos com a lima Reciproc R25 e } \\
\text { obturados com guta-percha e selante AH Plus } \\
\text { usando a técnica de onda contínua de } \\
\text { condensação. Em seguida as mostras foram } \\
\text { submetidas ao retratamento endodôntico com os } \\
\text { sistemas Reciproc, Reciproc Blue e XP-Endo } \\
\text { Shaper. O alargamento apical foi realizado com } \\
\text { Reciproc R40, Reciproc Blue R40 e BioRace } \\
\text { BR5. Cada amostra foi digitalizada após cada } \\
\text { procedimento endodôntico. O volume de } \\
\text { material de obturação remanescente foi } \\
\text { quantificado antes e após o alargamento apical. } \\
\text { Os volumes percentuais de redução da obturação } \\
\text { em relação aos canais instrumentados em ambos } \\
\text { os pontos de tempo (antes e depois do } \\
\text { alargamento apical) foram calculados. }\end{array}$ & $\begin{array}{l}\text { O instrumento XP-endo Shaper } \\
\text { mostrou uma maior porcentagem de } \\
\text { remoção da obturação, mas não } \\
\text { foram observadas diferenças } \\
\text { comparando Reciproc com o XP- } \\
\text { endo Shaper ou Reciproc Blue. O } \\
\text { aumento do alargamento apical } \\
\text { melhorou a remoção da obturação } \\
\text { em todos os grupos. Nenhum } \\
\text { instrumento foi capaz de manter } \\
\text { canais radiculares completamente } \\
\text { livres do material obturador. }\end{array}$ \\
\hline $\begin{array}{r}\text { Kirici et al. } \\
\text { (2020) }\end{array}$ & $\begin{array}{l}\text { Avaliar a quantidade de } \\
\text { material obturador, transporte } \\
\text { apical e formação de fissuras } \\
\text { após o retratamento em canais } \\
\text { radiculares curvos com os } \\
\text { sistemas Reciproc e Reciproc } \\
\text { Blue. }\end{array}$ & $\begin{array}{l}\text { Primeiros e segundos molares inferiores com } \\
\text { canais separados e ângulos entre } 20 \text { e } 40^{\circ} \text { foram } \\
\text { instrumentados com Reciproc R } 25 \text { e obturados } \\
\text { com cimento AhPlus. Em seguida os canais } \\
\text { foram retratados utilizando Reciproc ou } \\
\text { Reciproc Blue. Os canais foram retratados com } \\
\text { o instrumento R } 25 \text { e depois com o instrumento } \\
\text { R40 em ambos os grupos. O volume do material } \\
\text { obturador residual, transporte apical e formação } \\
\text { da fissura foram avaliadas usando imagem } \\
\text { micro-tomográfica computadorizada. }\end{array}$ & $\begin{array}{l}\text { O sistema Reciproc Blue é tão eficaz } \\
\text { quanto o Reciproc no retratamento } \\
\text { das raízes mesiais curvas de molares } \\
\text { inferiores. Novas fissuras } \\
\text { incompletas e completas foram } \\
\text { observadas em ambos os grupos. Os } \\
\text { valores de transporte do canal apical } \\
\text { foram significativamente maiores } \\
\text { para o Reciproc a } 1 \mathrm{~mm} \text { do ápice, } \\
\text { não havendo diferença com o } \\
\text { Reciproc Blue nos outros níveis do } \\
\text { canal. }\end{array}$ \\
\hline $\begin{array}{l}\text { Romeiro et al. } \\
\qquad(2019)\end{array}$ & $\begin{array}{l}\text { Comparar o material } \\
\text { obturador de canal radicular } \\
\text { remanescente, remoção de } \\
\text { dentina, transporte apical e } \\
\text { extrusão apical de detritos } \\
\text { após o retratamento de canais } \\
\text { obturados com cimentos } \\
\text { biocerâmicos ou à base de }\end{array}$ & $\begin{array}{l}\text { Molares inferiores com raízes mesiais } \\
\text { severamente curvas foram selecionados. As } \\
\text { amostras foram inicialmente instrumentadas } \\
\text { com a lima Reciproc R } 25 \text { e então dividido em } \\
\text { quatro grupos experimentais de acordo com o } \\
\text { cimento endodôntico e instrumento de } \\
\text { retratamento }(\mathrm{n}=15) \text { : BC Sealer / Reciproc, BC } \\
\text { Sealer / Reciproc Blue, AH Plus / Reciproc, e }\end{array}$ & $\begin{array}{l}\text { Reciproc Blue e Reciproc } \\
\text { apresentam a mesma eficácia na } \\
\text { remoção de material obturador, } \\
\text { independentemente do tipo de } \\
\text { cimento utilizado. Também não } \\
\text { houve diferença na quantidade de } \\
\text { material extrusionado ou no } \\
\text { transporte apical. O tempo de }\end{array}$ \\
\hline
\end{tabular}




\begin{tabular}{|c|c|c|c|}
\hline & $\begin{array}{l}\text { resina usando os instrumentos } \\
\text { Reciproc ou Reciproc Blue. }\end{array}$ & $\begin{array}{l}\text { AH Plus / Reciproc Blue. O tempo do } \\
\text { procedimento foi medido, e um tubo Eppendorf } \\
\text { foi usado em cada canal radicular para } \\
\text { armazenar a extrusão de detritos. As amostras } \\
\text { foram digitalizadas por micro-CT antes e após o } \\
\text { retratamento. O transporte apical, volume de } \\
\text { dentina e material obturador foram avaliados. }\end{array}$ & $\begin{array}{l}\text { retratamento para o Reciproc foi } \\
\text { significativamente } \\
\text { comparado a Reciproc Blue. }\end{array}$ \\
\hline $\begin{array}{c}\text { Bago et al. } \\
(2020)\end{array}$ & $\begin{array}{l}\text { Comparar a capacidade de } \\
\text { diferentes sistemas rotatórios e } \\
\text { limas reciprocantes no } \\
\text { retratamento em canais curvos } \\
\text { e avaliar a influência do } \\
\text { alargamento apical. }\end{array}$ & $\begin{array}{l}\text { Os canais foram preparados com o sistema } \\
\text { rotatório ProTaper Next, obturados com guta- } \\
\text { percha e selante a base de resina epóxi e } \\
\text { divididos em } 4 \text { grupos: Protaper Universal + } \\
\text { Protaper Gold, Reciproc Blue, Reciproc, } \\
\text { Waveone Gold. O Alargamento apical dos } \\
\text { grupos } 1,2 \text { e } 3 \text { foi realizado até o diâmetro } 40 \text {; e } \\
\text { no grupo } 4 \text { até o diâmetro } 35 \text {. }\end{array}$ & $\begin{array}{l}\text { Todos os quatro sistemas testados } \\
\text { foram igualmente eficazes na } \\
\text { remoção de material obturador dos } \\
\text { canais radiculares. O alargamento } \\
\text { apical melhorou a remoção do } \\
\text { material obturador remanescente. }\end{array}$ \\
\hline
\end{tabular}

Fonte: Autores.

Em relação aos artigos analisados, $89.6 \%$ dos estudos utilizaram limas RCB e $20.6 \%$ dos trabalhos usaram as PDR. No que tange aos estudos que analisaram as propriedades mecânicas das limas: a) RCB: 76.1\% avaliaram a resistência a fadiga cíclica, 23.8\% avaliaram a resistência torcional, e 14.2\% ambas as propriedades; b) PDR: 23.8\% avaliaram a fadiga cíclica e $14.2 \%$ a tensão torcional, e 14.2\% ambas as propriedades. Quanto aos estudos de remoção de material obturador pelas limas, $100 \%$ analisaram a eficácia das limas RCB, 22.2\% das limas PDR e 22.2\% comparando os instrumentos RCB e PDR.

Os principais achados das pesquisas foram:

I- Os instrumentos RCB (R25 ou R40) apresentam um maior número de ciclos até à fratura do que os instrumentos Reciproc (R25 ou R40), WaveOne Gold, HyFlex EDM, OneShape e 2Shape, independente do ângulo de curvatura dos canais artificiais $\left(45^{\circ}, 60^{\circ}, 80^{\circ}, 90^{\circ}\right)$ e do tipo de acionamento dos motores (elétrico, a ar ou controlado por torque) (Inan et al., 2018; Silva et al., 2018: Alcalde et al., 2018).

II- O instrumento PDR apresentou maior resistência à fadiga cíclica e maior ângulo de rotação em ensaios de torção comparado a outros instrumentos reciprocantes como a Reciproc, RCB, WaveOne Gold e Unicone. No entanto, a PDR apresentou menor resistência torcional em relação a esses instrumentos (De Menezes et al., 2017; Alcalde et al., 2017; Silva et al., 2018: Alcalde et al., 2018).

III- Os instrumentos RCB e PDR são eficazes na remoção do material obturador, mas nenhum dos sistemas analisados remove completamente o material obturador (Rodrigues et al., 2016; Bago et al., 2019; Borges et al., 2019; De Deus et al., 2019; Serefoglu et al., 2020).

\section{Discussão}

\section{Propriedades mecânicas da Reciproc Blue e ProDesign $R$}

A lima RCB apresenta tratamento térmico "blue", tecnologia baseada no arrefecimento e aquecimento da liga que lhe permite o controle sobre as temperaturas de transição, responsável pelo aumento da flexibilidade do instrumento, tornando assim o procedimento endodôntico mais seguro, permitindo melhor manutenção da curvatura do canal radicular e diminuindo o risco de fratura dos instrumentos (Topçuoğlu \& Topçuoğlu, 2017). A importância do tratamento térmico da RCB está evidente na maioria dos trabalhos que avaliaram comparativamente as propriedades mecânicas e de resistência desse instrumento. Em estudos que avaliaram o sistema RCB em face ao sistema Reciproc (M-Wire) evidenciaram que as limas com tratamento térmico blue demoraram mais tempo para fraturar (Keskin et al., 2017; Topçuoğlu \& Topçuoğlu, 2017), corroborando que as ligas de Ni-Ti com tratamento blue que apresentam maior resistência à fadiga cíclica em relação as limas Reciproc, que são feitas de ligas de Ni-Ti com tecnologia M-Wire. Nota-se melhor desempenho das limas produzidas com tratamento blue em comparação 
com outros instrumentos com diferentes tratamentos térmico como a WaveOne Gold (fabricada de liga de Ni-Ti Gold) (Keskin et al., 2017; Gündoğar \& Özyürek, 2017; Silva et al., 2018; Alcalde et al., 2018) e a HyFlex EDM (Gündoğar \& Özyürek, 2017). $\mathrm{O}$ instrumento X1 Blue, que apresenta ligas com tratamento térmico de superfície Blue com controle de memória, apresentou resistência similar a RCB (Inan et al., 2019).

Relativamente à análise da resistência torcional, o instrumento RCB apresentou deflexão angular até à fratura maior do que a Reciproc (Almeida et al., 2019), contudo uma menor resistência à torção até a fratura. Assim, na análise de fatores envolvidos na fratura de um instrumento, deve-se considerar tanto a fadiga cíclica quanto a fadiga torcional do instrumento, pois são parâmetros importantes para aumentar a segurança na utilização desses instrumentos.

O sistema PDR é produzido por ligas de Ni-Ti submetidos ao tratamento térmico de memória controlada CM-Wire, que favorece uma melhor distribuição da estrutura cristalina da liga, permitindo que estes instrumentos se encontrem na fase martensítica à temperatura ambiente. Este sistema, quando submetido a testes da flexibilidade e de resistência à fadiga cíclica, exibiu maior resistência do que os instrumentos Reciproc (Alcalde et al., 2017), Unicone (Silva et al., 2016), WaveOne Gold (Alcalde et al., 2017; Alcalde et al., 2018; De Menezes et al., 2017) e Reciproc Blue (Alcalde et al., 2018). Além disso, Silva et al. (2016) demonstraram que a PDR não tratada termicamente apresentou menor tempo até à fratura do instrumento em comparação com a PDR tratada. Essas referências evidenciam que o tratamento especial de controle de memória da liga das PDR é uma condição determinante para obter limas mais flexíveis e resistentes à fadiga cíclica. Além disso, análises comparativas da resistência à fadiga cíclica e torcional dos sistemas RCB e PDR mostraram que a Lima PDR apresenta maior tempo para fratura e maior rotação angular, mas menor carga de torque (Alcalde et al., 2018; Silva et al., 2018). No que se refere a ProDesign Logic, que possui o mesmo tamanho, desenho e secção transversal e diferente sentido da lâmina de corte (sentido horário) em relação à PDR (movimento anti-horário), apresentou maior número de ciclos até a fratura (De Menezes et al. 2017).

O tratamento térmico é um dos fatores essenciais para a maior flexibilidade dos instrumentos endodônticos, contudo outros fatores têm atuação importante nas propriedades mecânicas das limas. Em estudos comparativos da lima RCB com outros instrumentos, evidenciou-se que a resistência à fadiga cíclica é maior nas limas utilizadas no movimento reciprocante em relação às limas com movimentos rotatórios, como a One Shape (Gündoğar M \& Özyürek, 2017), 2Shape (Özyürek et al., 2018) e HyFlex ED (Gündoğar M \& Özyürek, 2017; Özyürek et al., 2018).

O diâmetro e a conicidade também influenciam nas propriedades mecânicas dos instrumentos de Ni-Ti, em especial em canais curvos. Apesar de todos os instrumentos analisados nesta revisão apresentarem o mesmo diâmetro em D0 (0.025 mm), dispunham de conicidades diferentes. A análise em destaque dos estudos envolvendo as limas PDR e RCB que apresentam respectivamente conicidades de $0.06 \mathrm{~mm} / \mathrm{mm}$ e de $0.08 \mathrm{~mm} / \mathrm{mm}$ (Silva et al., 2018; Alcalde et al., 2018), observou-se que o instrumento PDR foi mais resistente à fratura. Essa diferença no taper seria um fator a ser considerado para explicar a rigidez de um instrumento, influenciando assim nos valores do número de ciclos para fratura por fadiga cíclica (Yao et al., 2006).

Da mesma maneira, o design da secção transversal também afetou na flexibilidade e fadiga dos instrumentos. O instrumento PDR tem secção transversal em formato em S-modificado e a RCB, em formato S. Estudo realizado por Silva et al. (2018) demonstrou que o instrumento PDR, que tem menor área de seç̧ão transversal comparativamente ao WaveOne Gold (formato de paralelograma) e ao RCB, e apresentou maior resistência à fadiga cíclica. O instrumento WaveOne Gold apresenta maior secção transversal, e estudos mostraram que instrumentos com maior área de secção transversal têm maior rigidez flexural e torsional (Zhang et al., 2010; Baek et al., 2011) e, portanto, menor resistência à fadiga cíclica. Outra questão a ser considerada em relação à fratura de instrumentos é o ângulo e o raio de curvatura dos canais radiculares. Segundo Pruett et al. (1997) os níveis de tensão induzidos por curvaturas com menos de $5 \mathrm{~mm}$ de raio e ângulo de $30^{\circ}$ não resultam em separação do instrumento. Adıgüzel e Capar (2017) analisaram a resistência à fadiga cíclica de dois instrumentos reciprocantes de Ni-Ti, WaveOne e WaveOne Gold com diferentes tamanhos e diferentes ângulo de curvatura. A WaveOne Gold diferencia-se da WaveOne pelo 
tratamento térmico da liga (GOLD vs M-Wire) e secção transversal (paralelogramo vs triangular). Esses autores mostraram que o sistema WaveOne Gold é duas vezes mais resistente que a WaveOne em canais com ângulo de curvatura de $60^{\circ}$, e três vezes mais resistentes em canais com ângulo de curvatura de $90^{\circ}$. Para além disso, os estudos analisados nesta revisão realizaram testes de fadiga cíclica e resistência torcional em canais radiculares artificiais com diferentes ângulos e posição de curvatura. A maioria dos estudos optou pelos estudos em canais artificiais com ângulo de curvatura de $60^{\circ}$ e raio de $5 \mathrm{~mm}$, e observou-se a influência do tratamento térmico e outras características mecânicas dos instrumentos de forma positiva na resistência à fratura dos sistemas RCB e PDR, e mais ainda em estudos realizados em canais severamente curvos (Özyürek et al., 2018; Inan et al., 2019; Silva et al., 2018).

\section{Reciproc Blue e ProDesign R no Retratamento endodôntico}

Os trabalhos analisados que estudaram o comportamento dos instrumentos de Ni-Ti RCB e PDR no momento da fratura, seja por fadiga cíclica ou por torção, foram realizados maioritariamente em ensaios de preparo do canal radicular, ou seja, sem material obturador. A remoção máxima de material obturador é essencial para o sucesso do retratamento endodôntico.

Os sistemas reciprocantes RCB e PDR são indicados, de acordo com os respectivos fabricantes, para uso, tanto no preparo primário do canal radicular, como em procedimentos de retratamento endodôntico. Zuolo et al. (2013) demonstraram que os instrumentos que atuam por meio de movimento reciprocante foram mais rápidos e eficazes no processo de remoção de cimento e de guta-percha dos canais radiculares durante o retratamento em relação a técnicas rotatórias e manuais. Como já descrito, essa eficiência também se deve às suas características de elasticidade e resistência, o que possibilitaria uma redução de fraturas durante o procedimento de desobstrução de canais radiculares curvos.

A incidência de deformações e fraturas em instrumentos Reciproc (M-Wire) após o uso clínico foi analisada tanto no preparo de canais radiculares como na remoção de material obturador e posterior reparação dos canais (Plotino et al., 2015). O instrumento Reciproc R25 apresentou uma baixa taxa de fratura, sendo a ocorrência de deformações observada com mais frequência em casos de retratamentos do que em tratamentos primários, possivelmente devido à maior pressão exercida para remoção do material obturador nesses casos. Após análise pormenorizada dos artigos incluídos na presente revisão que realizaram estudo comparativo entre diferentes sistemas (Rodrigues et al., 2016; Bago et al., 2019; Borges et al., 2019) constatouse que não foi descrita qualquer deformação ou fratura nos instrumentos utilizados, quer em liga do tipo M-wire (Reciproc), CMWire (ProDesign R, ProDesign Logic), com tratamento Blue (Reciproc Blue), tratamento GOLD (WaveOne Gold) ou sem tratamento (Mtwo, Protaper Universal).

Outro aspecto importante a ser explorado em relação as limas do estudo é que foram projetados seguindo o princípio de lima única e de uso único, proposto por Yared (2008), isto é, apenas um instrumento é necessário para preparar um canal radicular, reduzindo assim os riscos de fratura, contaminação cruzada e número de instrumentos necessários para o preparo do canal (Haïkel et al., 1999). No entanto, considerando o custo econômico de um procedimento, trabalhos analisaram a viabilidade da aplicação clínica da reutilização limitada desses instrumentos (Bueno et al., 2017; Pirani et al., 2014). Comparando-se as propriedades metalúrgicas de instrumentos Reciproc e RCB novos e usados em canais artificiais, foi observado que a RCB usada apresentou resistência à fadiga cíclica similar à Reciproc nova (Generali et al., 2020). Na análise do efeito do uso sequencial da RCB durante o retratamento (Serefoglu et al., 2010), foi demonstrado, por teste de fadiga cíclica e tempo de trabalho, que o instrumento pode ser usado com segurança na remoção de material obturador de até dois molares.

As ligas dos instrumentos RCB e PDR, devido às características de rigidez e de elasticidade que apresentam, desempenharam papel importante no acesso progressivo a canais radiculares severamente curvos e também na recuperação da patência ou permeabilidade apical durante o procedimento de retratamento endodôntico (De Deus et al., 2019). Os estudos analisados nesta revisão mostraram que os instrumentos RCB e PDR foram eficientes na remoção de grande quantidade de 
material obturador, mas nenhum foi capaz de remover completamente o material do canal radicular (Rodrigues et al., 2016; Bago et al., 2019; Borges et al., 2019), o que está de acordo com vários resultados da literatura (Martins et al., 2017; Crozeta et al., 2016; Takahashi et al., 2019). O alargamento apical (RCB R25 para RCB R40) contribuiu para remoção do material obturador dos canais radiculares (De Deus et al., 2019), sendo esse efeito semelhante para todos os sistemas estudados. Além disso, mesmo utilizando como material obturador guta-percha, associada a diferentes cimentos, à base de resina epóxi (incluindo AH-Plus) (Serefoglu et al., 2010; De Deus et al., 2019), à base de óxido de zinco e eugenol (Rodrigues et al., 2016), ou biocerâmicos (Romeiro et al., 2020), não houve diferenças significativas na quantidade de material removido pelos instrumentos.

Para, além disso, um estudo indicou que a remoção do material obturador, em combinação com diferentes instrumentos (RCB R25 seguida de RCB R40; PDR R25 seguida de PDR R35; WaveOne Gold R25 seguida de WaveOne Gold R35) e complementadas com abordagens adicionais (irrigação ultrassônica passiva, instrumento XP-Endo Shaper, lima H) (Borges et al., 2019), melhora a limpeza do canal radicular. O uso de instrumentos auxiliares para complementar a limpeza tem sido considerado mais uma estratégia para superar o desafio da limpeza dos canais radiculares, no processo de retratamento endodôntico (Bao et al., 2017; Silva et al., 2018).

\section{Conclusão}

Com base nos artigos estudados, foi possível constatar que a hipótese desta revisão foi verificada. Embora seja notável a importância das propriedades mecânicas e tratamento térmico das ligas de Ni-Ti no comportamento dos instrumentos endodônticos RCB e PDR, estes sistemas mostraram desempenhos semelhantes na remoção de material obturador dos canais quando comparados a outros instrumentos. Ambos os sistemas foram eficientes na remoção de grande quantidade de material obturador, mas, com qualquer deles, não se verificou uma limpeza completa dos canais radiculares.

\section{Referências}

Adıgüzel, M., \& Capar, I. D. (2017). Comparison of cyclic fatigue resistance of WaveOne and WaveOne Gold small, primary, and large instruments. Journal of endodontics, 43(4), 623-627.

Ahn, S. Y., Kim, H. C., \& Kim, E. (2016). Kinematic effects of nickel-titanium instruments with reciprocating or continuous rotation motion: a systematic review of in vitro studies. Journal of endodontics, 42(7), 1009-1017.

Alcalde, M. P., Duarte, M. A. H., Bramante, C. M., de Vasconselos, B. C., Tanomaru-Filho, M., Guerreiro-Tanomaru, J. M., \& Vivan, R. R. (2018). Cyclic fatigue and torsional strength of three different thermally treated reciprocating nickel-titanium instruments. Clinical oral investigations, 22(4), $1865-1871$.

Alcalde, M. P., Tanomaru-Filho, M., Bramante, C. M., Duarte, M. A. H., Guerreiro-Tanomaru, J. M., Camilo-Pinto, J., \& Vivan, R. R. (2017). Cyclic and torsional fatigue resistance of reciprocating single files manufactured by different nickel-titanium alloys. Journal of endodontics, 43(7), 1186-1191.

Almeida, G. C., Guimarães, L. C., Resende, P. D., Buono, V. T. L., Peixoto, I. F. C., \& Viana, A. C. D. (2019). Torsional behaviour of Reciproc and Reciproc blue instruments associated with their martensitic transformation temperatures. International endodontic journal, 52(12), 1768-1772.

Al-Obaida, M. I., Merdad, K., Alanazi, M. S., Altwaijry, H., AlFaraj, M., Alkhamis, A. A., \& Al-Madi, E. M. (2019). Comparison of cyclic fatigue resistance of 5 heat-treated nickel-titanium reciprocating systems in canals with single and double curvatures. Journal of endodontics, 45(10), $1237-1241$.

Baek, S. H., Lee, C. J., Versluis, A., Kim, B. M., Lee, W., \& Kim, H. C. (2011). Comparison of torsional stiffness of nickel-titanium rotary files with different geometric characteristics. Journal of endodontics, 37(9), 1283-1286.

Bago, I., Suk, M., Katić, M., Gabrić, D., \& Anić, I. (2019). Comparison of the effectiveness of various rotary and reciprocating systems with different surface treatments to remove gutta-percha and an epoxy resin-based sealer from straight root canals. International endodontic journal, 52(1), 105-113.

Bao, P., Shen, Y., Lin, J., \& Haapasalo, M. (2017). In vitro efficacy of XP-endo Finisher with 2 different protocols on biofilm removal from apical root canals. Journal of endodontics, 43(2), 321-325.

Borges, M. M., Duque, J. A., Zancan, R. F., Vivan, R. R., Bernardes, R. A., \& Duarte, M. A. (2019). Efficacy of reciprocating systems for removing root filling material plus complementary cleaning methods in flattened canals: Microtomography and scanning electron microscopy study. Microscopy research and technique, 82(7), 1057-1064.

Bueno, C. S. P., de Oliveira, D. P., Pelegrine, R. A., Fontana, C. E., Rocha, D. G. P., \& da Silveira Bueno, C. E. (2017). Fracture incidence of WaveOne and Reciproc files during root canal preparation of up to 3 posterior teeth: a prospective clinical study. Journal of endodontics, 43(5), 705-708. 
Crozeta, B. M., Silva-Sousa, Y. T. C., Leoni, G. B., Mazzi-Chaves, J. F., Fantinato, T., Baratto-Filho, F., \& Sousa-Neto, M. D. (2016). Micro-computed tomography study of filling material removal from oval-shaped canals by using rotary, reciprocating, and adaptive motion systems. Journal of endodontics, 42(5), 793-797.

De Menezes, S. E. A. C., Batista, S. M., Lira, J. O. P., \& de Melo Monteiro, G. Q. (2017). Cyclic fatigue resistance of WaveOne Gold, ProDesign R and ProDesign Logic files in curved canals in vitro. Iranian endodontic journal, 12(4), 468.

De-Deus, G., Silva, E. J. N. L., Vieira, V. T. L., Belladonna, F. G., Elias, C. N., Plotino, G., \& Grande, N. M. (2017). Blue thermomechanical treatment optimizes fatigue resistance and flexibility of the Reciproc files. Journal of endodontics, 43(3), 462-466.

De-Deus, G., Belladonna, F. G., Zuolo, A. S., Simões-Carvalho, M., Santos, C. B., Oliveira, D. S., \& Silva, E. J. N. L. (2019). Effectiveness of Reciproc Blue in removing canal filling material and regaining apical patency. International endodontic journal, 52(2), 250-257.

De-Deus, G., Belladonna, F. G., de Siqueira Zuolo, A., Cavalcante, D. M., Carvalho, M. S., Marinho, A., \& Silva, E. J. N. L. (2019). 3-Dimensional ability assessment in removing root filling material from pair-matched oval-shaped canals using thermal-treated instruments. Journal of endodontics, 45(9), 1135-1141.

Del Fabbro, M., Afrashtehfar, K. I., Corbella, S., El-Kabbaney, A., Perondi, I., \& Taschieri, S. (2018). In vivo and in vitro effectiveness of rotary nickel-titanium vs manual stainless-steel instruments for root canal therapy: systematic review and meta-analysis. Journal of Evidence Based Dental Practice, 18(1), 59-69.

Generali, L., Puddu, P., Borghi, A., Brancolini, S., Lusvarghi, L., Bolelli, G., \& Pedullà, E. (2020). Mechanical properties and metallurgical features of new and ex vivo used Reciproc Blue and Reciproc. International endodontic journal, 53(2), 250-264.

Gündoğar, M., \& Özyürek, T. (2017). Cyclic fatigue resistance of OneShape, HyFlex EDM, WaveOne Gold, and Reciproc Blue nickel-titanium instruments. Journal of endodontics, 43(7), 1192-1196.

Haikel, Y., Serfaty, R., Bateman, G., Senger, B., \& Allemann, C. (1999). Dynamic and cyclic fatigue of engine-driven rotary nickel-titanium endodontic instruments. Journal of endodontics, 25(6), 434-440.

Iacono, F., Pirani, C., Arias, A., Josè, C., Generali, L., Gandolfi, M. G., \& Prati, C. (2019). Impact of a modified motion on the fatigue life of NiTi reciprocating instruments: a Weibull analysis. Clinical oral investigations, 23(7), 3095-3102.

Inan, U., Keskin, C., Yilmaz, Ö. S., \& Baş, G. (2019). Cyclic fatigue of Reciproc Blue and Reciproc instruments exposed to intracanal temperature in simulated severe apical curvature. Clinical oral investigations, 23(5), 2077-2082.

Keskin, C., Inan, U., Demiral, M., \& Keleş, A. (2017). Cyclic fatigue resistance of Reciproc Blue, Reciproc, and WaveOne Gold reciprocating instruments. Journal of endodontics, 43(8), 1360-1363.

Klymus, M. E., Alcalde, M. P., Vivan, R. R., Só, M. V. R., de Vasconselos, B. C., \& Duarte, M. A. H. (2019). Effect of temperature on the cyclic fatigue resistance of thermally treated reciprocating instruments. Clinical oral investigations, 23(7), 3047-3052.

Kırıc1, D., Demirbuga, S., \& Karataş, E. (2020). Micro-computed tomographic assessment of the residual filling volume, apical transportation, and crack formation after retreatment with Reciproc and Reciproc Blue Systems in curved root canals. Journal of endodontics, 46(2), 238-243.

Limoeiro, A. G., Braitt, A. H., Machado, A. S., Bueno, C. E., Fontana, C. E., Freire, L. G., \& De Martin, A. (2021). Micro-computed tomography evaluation of filling material removal by three reciprocating systems with different thermal treatments. Giornale Italiano di Endodonzia, (Early View).

Martins, M. P., Duarte, M. A. H., Cavenago, B. C., Kato, A. S., \& da Silveira Bueno, C. E. (2017). Effectiveness of the protaper next and reciproc systems in removing root canal filling material with sonic or ultrasonic irrigation: a micro-computed tomographic study. Journal of endodontics, 43(3), 467-471.

Nascimento, W. M., da Silva Limoeiro, A. G., Moraes, M. M., Campos, D. D. L., Drumond, J. P., Maeda, W., . \& Frozoni, M. (2021). Reduction in Enteroccocus faecalis counts produced by three file systems in severely curved canals. Research, Society and Development, 10(2).

Nevares, G., Diana, S., Freire, L. G., Romeiro, K., Fogel, H. M., Dos Santos, M., \& Cunha, R. S. (2016). Efficacy of ProTaper NEXT compared with Reciproc in removing obturation material from severely curved root canals: a micro-computed tomography study. Journal of endodontics, 42(5), 803-808.

Özyürek, T., Gündoğar, M., Uslu, G., Yılmaz, K., Staffoli, S., Grande, N. M., \& Polimeni, A. (2018). Cyclic fatigue resistances of Hyflex EDM, WaveOne gold, Reciproc blue and 2shape NiTi rotary files in different artificial canals. Odontology, 106(4), 408-413.

Pirani, C., Paolucci, A., Ruggeri, O., Bossù, M., Polimeni, A., Gatto, M. R. A., \& Prati, C. (2014). Wear and metallographic analysis of WaveOne and reciproc NiTi instruments before and after three uses in root canals. Scanning The Journal of Scanning Microscopies, 36(5), 517-525.

Plotino, G., Grande, N. M., \& Porciani, P. F. (2015). Deformation and fracture incidence of R eciproc instruments: a clinical evaluation. International endodontic journal, 48(2), 199-205.

Plotino, G., Grande, N. M., Testarelli, L., Gambarini, G., Castagnola, R., Rossetti, A., \& Fortunato, L. (2018). Cyclic fatigue of Reciproc and Reciproc Blue nickel-titanium reciprocating files at different environmental temperatures. Journal of endodontics, 44(10), 1549-1552.

Pruett, J. P., Clement, D. J., \& Carnes Jr, D. L. (1997). Cyclic fatigue testing of nickel-titanium endodontic instruments. Journal of endodontics, 23(2), 77-85.

Rios, AM., Villela, A. M., Cunha, R. S., Velasco, R. C., De Martin, A. S., Kato, A. S., \& da Silveira Bueno, C. E. (2014). Efficacy of 2 reciprocating systems compared with a rotary retreatment system for gutta-percha removal. Journal of endodontics, 40(4), 543-546.

Rôças, I. N., Provenzano, J. C., Neves, M. A., \& Siqueira Jr, J. F. (2016). Disinfecting effects of rotary instrumentation with either $2.5 \%$ sodium hypochlorite or $2 \%$ chlorhexidine as the main irrigant: a randomized clinical study. Journal of endodontics, 42(6), 943-947.

Rodrigues, C. T., Duarte, M. A. H., de Almeida, M. M., de Andrade, F. B., \& Bernardineli, N. (2016). Efficacy of CM-Wire, M-Wire, and Nickel-Titanium Instruments for Removing Filling Material from Curved Root Canals: A Micro-Computed Tomography Study. Journal of endodontics, 42(11), 1651-1655. 
Research, Society and Development, v. 10, n. 6, e4010615443, 2021

(CC BY 4.0) | ISSN 2525-3409 | DOI: http://dx.doi.org/10.33448/rsd-v10i6.15443

Romeiro, K., de Almeida, A., Cassimiro, M., Gominho, L., Dantas, E., Chagas, N., \& Albuquerque, D. (2020). Reciproc and Reciproc Blue in the removal of bioceramic and resin-based sealers in retreatment procedures. Clinical oral investigations, 24(1), 405-416.

Schneider, S. W. (1971). A comparison of canal preparations in straight and curved root canals. Oral surgery, Oral medicine, Oral pathology, 32(2), $271-275$.

Serefoglu, B., Kurt, S. M., Kaval, M. E., Güneri, P., Demirci, G. K., \& Çalı̧̧kan, M. K. (2020). Cyclic Fatigue Resistance of Multiused Reciproc Blue Instruments during Retreatment Procedure. Journal of endodontics, 46(2), 277-282.

Silva, E. J. N. L., Rodrigues, C., Vieira, V. T. L., Belladonna, F. G., De-Deus, G., \& Lopes, H. P. (2016). Bending resistance and cyclic fatigue of a new heattreated reciprocating instrument. Scanning, 38(6), 837-841.

Silva, E. J. N. L., Vieira, V. T. L., Hecksher, F., dos Santos Oliveira, M. R. S., dos Santos Antunes, H., \& Moreira, E. J. L. (2018). Cyclic fatigue using severely curved canals and torsional resistance of thermally treated reciprocating instruments. Clinical oral investigations, 22(7), 2633-2638.

Silva, E. J. N. L., Vieira, V. T. L., Gabina, T. T. G., dos Santos Antunes, H., Lopes, H. P., \& De-Deus, G. (2019). The impact of using a pneumatic contra-angle device on the lifespan of M-Wire-and Blue-treated instruments. Clinical oral investigations, 23(2), 617-621.

Takahashi, C. M., Cunha, R. S., De Martin, A. S., Fontana, C. E., Silveira, C. F. M., \& da Silveira Bueno, C. E. (2009). In vitro evaluation of the effectiveness of ProTaper universal rotary retreatment system for gutta-percha removal with or without a solvent. Journal of Endodontics, 35(11), 1580-1583.

Topçuoğlu, H. S., \& Topçuoğlu, G. (2017). Cyclic fatigue resistance of Reciproc Blue and Reciproc files in an S-shaped canal. Journal of endodontics, 43(10), $1679-1682$

Topçuoğlu, H. S., Demirbuga, S., Düzgün, S., \& Topçuoğlu, G. (2018). Cyclic fatigue resistance of new reciprocating files (Reciproc Blue, WaveOne Gold, and SmartTrack) in two different curved canals. Journal of investigative and clinical dentistry, 9(3), e12344.

Torabinejad, M., Corr, R., Handysides, R., \& Shabahang, S. (2009). Outcomes of nonsurgical retreatment and endodontic surgery: a systematic review. Journal of endodontics, 35(7), 930-937.

Yao, J. H., Schwartz, S. A., \& Beeson, T. J. (2006). Cyclic fatigue of three types of rotary nickel-titanium files in a dynamic model. Journal of endodontics, 32(1), 55-57.

Yared, G. (2008). Canal preparation using only one Ni-Ti rotary instrument: preliminary observations. International endodontic journal, 41(4), 339-344.

Zhang, E. W., Cheung, G. S., \& Zheng, Y. F. (2010). Influence of cross-sectional design and dimension on mechanical behavior of nickel-titanium instruments under torsion and bending: a numerical analysis. Journal of endodontics, 36(8), 1394-1398.

Zuolo, A. S., Mello Jr, J. E., Cunha, R. S., Zuolo, M. L., \& Bueno, C. E. S. (2013). Efficacy of reciprocating and rotary techniques for removing filling material during root canal retreatment. International Endodontic Journal, 46(10), 947-953.

Zupanc, J., Vahdat-Pajouh, N., \& Schäfer, E. (2018). New thermomechanically treated NiTi alloys-a review. International endodontic journal, 51(10), 10881103 . 